\section{Only the smile is left}

SIR-The 30 June 1988 issue of Nature contains a "Scientific Paper" describing unusual results. In it, Davenas et al. ' $^{\text {claim }}$ that images of molecules may be imposed on solvent such that when the original molecules are no longer present (for example, by diluting the solution sufficiently) their reactivity nevertheless persists. Specific antibodies, antigens, enzymes, ionophores and simple amines were claimed to exhibit such 'Cheshire cat' ${ }^{2}$ phenomena. Because the assay used by Davenas et al. is closely analogous to one regularly used in our laboratory, it was simple for us to test the generalizability of their claims. We observed no results such as theirs. Evidently, whatever may have generated their own data is not readily reproduced. We therefore see no basis as yet for concluding that the chemical data accumulated over two centuries are in error, and that Davenas et al. have discovered a new chemical principle.

Rat basophilic leukaemia cells - a neoplastic line of mucosal mast cells of the $2 \mathrm{H} 3(\mathrm{HR}+)$ line $^{3}-$ were grown in stationary flasks and after harvesting were mixed with ${ }^{3} \mathrm{H}$-labelled- 5 hydroxytryptamine (5HT) and mouse anti-dinitrophenyl immunoglobulin $\mathrm{E}$ (IgE). The mixture was distributed on 24-well polystyrene plates and incubated for 16 hours. After washing, the adherent cells were challenged with either purified rabbit anti-mouse IgE or dinitrophenylated bovine serum albumin ${ }^{4}$. In preparing sequential dilutions of the antibody and antigen, care was taken to vortex each dilution for approximately 12 seconds because Davenas et al. claimed this was important ${ }^{1}$. The dilutions were prepared and coded by one of us (H.M.); the assay performed and data analysed by the other (S.D.). Our results are shown in Fig. 1. No release of 5-HT was observed at high dilutions. It is apparent that the phenomenon described by Davenas et al. is not readily generalizable even to a closely related system.

It is reasonable to ask whether the observations of Davenas et al. should have been published by Nature. We think not. One of us (H.M.) reviewed their paper at the request of Nature in April 1987, and urged that the findings be checked by one or more laboratories chosen by the editor. Instead, Dr Benveniste made his own choice, and Nature decided to publish the report and then to despatch an international investigative team consisting of the editor, a magician and a scientist, none of whom has experience in the relevant field. Their report ${ }^{5}$ provides no support for the published claims and will dismay serious scientists: it adds to the circus atmosphere engendered by the publication of the original paper. ("Homoeo- pathic enthusiasts are rejoicing while scientists scratch their heads..."; "Homoeopathy finds scientific support" ${ }^{\text {") }}$ ) We believe that the approach chosen by Nature is regrettable. We feel that all ideas no matter how revolutionary, deserve to be heard. However, when new data are proffered that grossly conflict with vast amounts of earlier, welldocumented and easily replicated data, a different editorial standard is required. Before the imprimatur inherent in publishing them in a leading scientific journal is granted, the new results must be reproducible by disinterested individuals familiar with the field. That is a fundamental principle of scientific objectivity. It's a shame really. It still takes a full teaspoon of sugar to sweeten our tea.

Henry Metzger

Building 10, $\mathrm{Rm}$ 9N258,

StePHen C. DrEskin

NIAMS, National Institutes of Health,

Bethesda, Maryland 20892, USA

1. Davenas, E. et al. Nature 333, 816-818 (1988)

2. Carroll, L. Alice in Wonderland (Grosset and Dunlap, New/ York, 1987).

. Barsumian et al. Eur. J. Immunol. 11, 317-323 (1981).

. Maeyama et al. J. biol. Chem. 261, 2583-2592 (1986).

Maddox, J. et al. Nature 334, 287 (1988)

6. New Scientist 14 July 1988 p. 39.

7. Newsweek 25 July, 1988 p. 66.

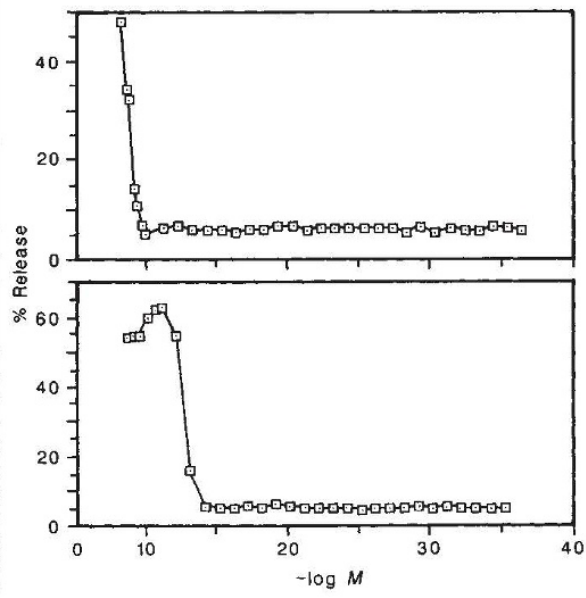

Release of incorporated ${ }^{3} \mathrm{H}-5 \mathrm{HT}$ from tumour basophils. Each specimen contained approximately $5 \times 10^{5}$ cells and $200 \mu \mathrm{l}$ medium (100 $\mathrm{mM} \mathrm{NaC} 1,5 \mathrm{mM} \mathrm{KC1}, 1 \mathrm{mM} \mathrm{CaC1}, 0.4 \mathrm{mM}$ $\mathrm{MgC1}_{2}, 5.6 \mathrm{mM}$ glucose, $25 \mathrm{mM} \mathrm{Na} \mathrm{m}_{2}$ PIPES, $0.1 \%$ bovine serum albumin, $p \mathrm{H} \mathrm{7.0)}$. The appropriate (coded) dilution of antibody $(a)$ or antigen $(b)$ was added and incubation continued for $45 \mathrm{~min}$. The supernatant was aspirated and counted; $250 \mu \mathrm{l}$ of $1 \%$ Triton X100 in phosphate buffered saline $X 3$ was used to release the cells and counted. Percent release was calculated as previously described ${ }^{4}$. The solid squares on each graph show the average for three buffer controls performed in duplicate for each set. All data are the means. The ranges of duplicates were $\pm 5 \%$ or less from the mean. Altogether there were 142 samples counted and all the data are shown.
SIR-In order that the controversial work of Davenas et al. should be considered credible, the reader must first be convinced that the data are genuine. Examination of the data represented in Table 1, however, convinces me that they are synthetic.

The results given are based on triplicate counts of approximately 80 cells and are expressed as the arithmetic mean and standard error. The standard errors vary between 0.5 and 4.8 with a median value of 1.5. This value is much less than that anticipated if normal chance variation in cell number were to have been observed. Even if no cell clumping occurred in the pipetted samples and the observers were wholly accurate, the value of the standard error is too small. In practice, this value will always be equal to or greater than the theoretical minimum standard error.

The most damning evidence is in their Fig. $1 b$. The number of basophils in the anti-IgG control wells, may be either greater or less than that observed in the untreated control wells, assuming that anti-IgG has no effect. It therefore follows that the "\% basophil degranulation" should be less than zero in approximately 30 of the 60 dilutions used. The absence of any such 'negative' degranulation figures and the overall distribution of the results is disturbing.

As it is obvious that the data presented in Table 1 and Figure 1 are not strictly derived by experiment, the credibility of the remainder of the paper must remain in doubt.

P.M. GAylarde Department of Dermatology,

Royal Free Hospital, London NW3, UK

SIR-J. Benveniste and co-workers recently reported extraordinary observations on the effects of extremely diluted antibody solutions. They found that aqueous solutions of anti-immunoglobulin $\mathrm{E}$ (anti-IgE) still retain an ability to cause degranulation of human basophils (a type of white blood cell with cell surface antibodies to $\mathrm{IgE}$ ) even when diluted to the point where there are no molecules of anti-IgE left in solution'. We suggest that these results are due to the production of reactive chemical species by cavitation during sample preparation.

As these authors state ${ }^{1}$, the "importance of agitation in the transmission of information was explored by pipetting dilutions up and down ten times and comparing with the usual 10 -s vortexing. Although the two processes resulted in the same dilution..., degranulation did not occur at high dilution after pipetting. Tensecond vortexing was the minimum time required.... So transmission of the information depended on vigorous agitation..."

The authors are apparently unaware of 